\title{
Prevalence and Sensitivity Patterns of Candidal Infections in Various Tertiary Care Health Subunits of Karachi
}

\author{
Rabeea Rizwan', Zahida Memon'1, Shehla Shaheen1, Faisal Afridi², Mubarak Zaib² \\ ${ }^{1}$ Department of Pharmacology, Ziauddin Medical College, Karachi, Pakistan \\ ${ }^{2}$ Department of Microbiology, Ziauddin University Hospital, Karachi, Pakistan \\ Email: rabeea_rizwan@hotmail.com,drshel2011@gmail.com
}

How to cite this paper: Rizwan, R., Memon, Z., Shaheen, S., Afridi, F. and Zaib, M. (2018) Prevalence and Sensitivity Patterns of Candidal Infections in Various Tertiary Care Health Subunits of Karach. International Journal of Clinical Medicine, 9, 645-659.

https://doi.org/10.4236/ijcm.2018.99054

Received: August 8, 2018

Accepted: August 26, 2018

Published: August 29, 2018

Copyright $\odot 2018$ by authors and Scientific Research Publishing Inc. This work is licensed under the Creative Commons Attribution International License (CC BY 4.0).

http://creativecommons.org/licenses/by/4.0/

(c) (i) Open Access

\begin{abstract}
Mostly candida resides as an opportunistic organism on epithelial surfaces of human being. However, under auspicious conditions can cause infections including serious life threatening invasive candidiasis with subsequent mortality particularly in immune deficit and hospitalized patients having co-morbids. Limited data are published on the prevalence of candidiasis, based on the researches conducted at few tertiary care settings which are not representing the overall disease burden in our country, Pakistan. Therefore, this study was conducted to evaluate the frequency and sensitivity patterns of candidiasis in our community. Methods: Out of total 1020 specimens, 130 clinical samples were identified as candidal positive, obtained from March to May 2018. These samples were isolated from vagina, oropharynx, urine, tracheal aspirates, pus, blood, tips of the intubations, wounds and fluids of the body cavities. Identification of candida, its species and antifungal sensitivity screening was done by Kirby Bauer's disk diffusion method according to CLSI guide lines' (M 44 A2 series, 2009). Results: A significant majority, 80 (61.5\%) of candidal strains were isolated from females with female to male ratio 8:5 and most of these isolates were obtained from high vaginal swabs (43.75\%). Four candidal species (Candida albicans $80 \%$, Candida tropicalis $10 \%$, Candida glabrata 9.2\% and Candida ciferrii $0.8 \%$ ) were isolated from all positive specimens. Maximum number of the positive samples 52 (40\%) were obtained from ICU patients. Sensitivity test of candidal positive samples revealed that commonly used azole antifungal drugs, fluconazole and voriconazole were highly resistant, with respective $57.7 \%$ and $70.8 \%$ resistance. Conclusion: Candidiasis is highly prevalent in our clinical set up and more frequently infecting females in comparison to males as most of the positive isolates were retrieved from HVS (high vaginal swabs). Still, C. albicans was found to be the most preva-
\end{abstract}


lent specie isolated among all candida samples. Our study also demonstrated that the resistance of most commonly prescribed antifungals, azoles have shown a rapid rise. Therefore, it is recommended that before prescription of antifungal drugs the clinicians should routinely recommend culture and sensitivity testing of samples taken from candida infected individuals.

\section{Keywords}

Candida, Candidiasis, Antifungal Drugs, Fluconazole, Voriconazole, C. albicans, Candidal Infection, Sensitivity, Resistance

\section{Introduction}

In daily life, human beings are commonly exposed to numerous microorganisms including fungi, among which some behave as normal commensals while other could be pathogenic. According to CDC (Centers for Disease Control), three wide classes of fungi are of concern, these are; 1) opportunistic, 2) community acquired 3) hospital acquired [1]. Candida, an ascomycetous yeast is one of the common fungi responsible for infecting individuals across the globe [2]. It is one of the normal floras of our body surfaces, acts as opportunistic organism which dwells in $70 \%$ of human population. Under certain suitable and privileged circumstances candida can infect through its resident areas like oropharynx, vagina, vulva, urethra, ears externa and nails. It can also invade the affected distorted epithelial surfaces by forming hyphae and can become the source of systemic infection. It usually causes recurrent infections in spite of prolonged antifungal therapy [3] [4] [5] [6].

Global warming is continuously rising that declines the temperature gradient between human and fungi which becomes one of the reasons of rise in fungal infections around the world [7]. Pakistan is a land of varied geographical features with extremes of seasonal variations seen across the country [8]. These geographical factors, such as hot and humid weather, heavy rain falls during monsoons and floods are thought to increase the risk of acquiring fungal infections among our population. It is usually associated with people of extreme ages, weakened or altered immunity, prolonged hospitalization and organ transplantation. Prolonged catheterization, use of chemotherapeutics, immunosuppressants, corticosteroids (topical/oral), and chronically ill individuals on polypharmacy are also associated risk factors for candidiasis. Since $19^{\text {th }}$ century, the incidence and prevalence rate of mycotic diseases is continuously rising in aforementioned high-risk population [9]. Candidiasis is now becoming a grave health related issue due to continuous rise of its high morbidity and mortality rates with passing time [9].

Routinely prescribed antifungal drugs for treating candidiasis included azoles, polyenes and echinocandins. Topographic variance has been observed in the 
occurrence rate and antifungal sensitivity of various candidal species as evident from the data of different countries and clinical setups around the world [10]. Therefore, this study was conducted at four subunits of a tertiary care hospital located in different areas of Karachi to evaluate the prevalence of candidiasis along with species identification and their antifungal resistance patterns in our community.

\section{Materials and Methods}

This prospective quasi experimental study was conducted at four subunits of a tertiary care, Ziauddin hospital located in different areas of Karachi, from March, 2018 to May, 2018. After approval from all competent committees including Ethical Review Committee of Ziauddin University, research work was initiated. After informed consent patients with signs and symptoms of fungal infections like white patchy and itchy skin or mucosal regions, additionally patients with their prolonged hospitalization and on prolonged antibiotics therapy presented with constant fatigue, recurrent infections of different regions of the body and whose laboratory test found candida positive, were included in this study. While those patients, didn't want to participate were excluded from the study. Their samples were taken from oropharynx (oral and throat swabs), blood, respiratory tract (sputum, tracheal secretions, nasopharyngeal aspirates, bronchoalveolar lavage), genitals (high vaginal swab), pus and urine for culture and sensitivity $(\mathrm{C} / \mathrm{S})$. All samples were processed in microbiology laboratory within 24 hours according to standard protocols. Patients' data was entered in electronic hospital software (LIS-Laboratory Information System).

\subsection{Methodology}

According to the standard procedures, yeast were identified by; BACTEC $9240^{\mathrm{TM}}$ for blood specimens, other specimens like vaginal swabs were gram stained and wet mounted. Germ tube test was also performed to identify hyphal yeast. Sabouraud dextrose agar media (SDA, Oxoid, UK) was prepared according to manufacturer's directions along with adding Chloramphenicol to inhibit bacterial growth on it. Candida was grown by streaking on media (SDA) as shown in Picture 1. After 48 hours of positive fungal growth, species identification was done on differential agar media, CHROME agar candida (Oxoid, UK) by incubating at $35^{\circ} \mathrm{C}$ for 2 days which showed different colored grown colonies of various candidal species (Picture 2). Species identification was further confirmed by API 20 C AUX method. At 0.5 MacFarland's, turbidity culture sensitivity test was done by 'Kirby Bauer's method and CLSI guide lines' (M - 44 A2 series, 2009) [11]. For this purpose, Muller Hinton Agar Media (MHA) was prepared according to company instructions (Oxoid, UK), along with adding $2 \%$ glucose and $0.5 \%$ Methylene blue in it. Antifungal discs of fluconazole $10 \mathrm{mcg}$ and voriconazole $1 \mathrm{mcg}$ (HiMedia, India) were placed on these media. After two days of incubation, zones of inhibition (ZIH) around each disc were noted to estimate the sensitivity patterns of respective antifungal drugs [12] [13]. 


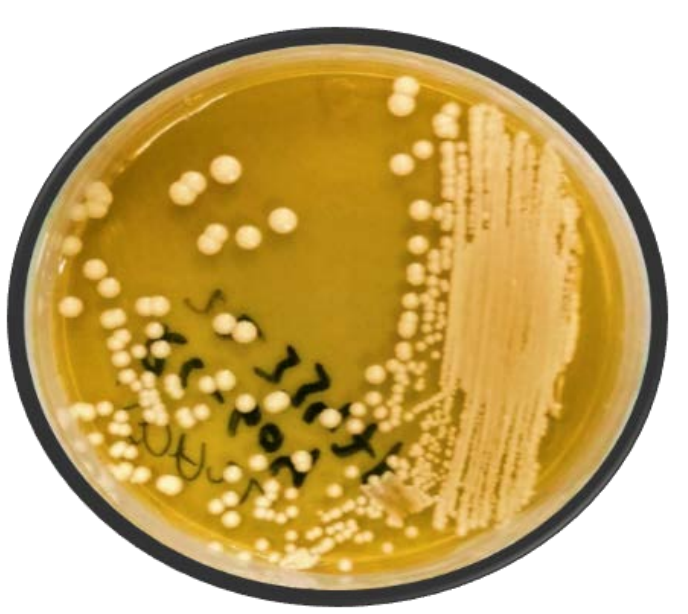

Picture 1. Candidal positive, white velvety colonies of a specimen grown on Sabouraud dextrose agar media.

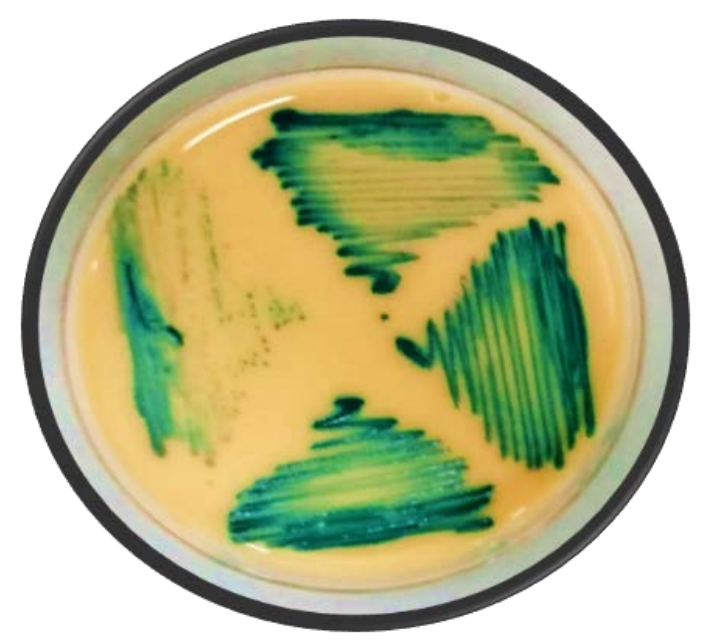

Picture 2. Green colored colonies showing Candida albicans positive samples, grown on differential CHROMagar media.

\subsection{Data Analysis}

The sample size was calculated by Sealed Envelope calculator version 2012 .

The calculated sample size was 64 but for this research 100 samples by consecutive sampling technique were taken according to the inclusion and exclusion criteria.

All collected data was entered and analyzed by using SPSS (Statistical Package for the Social Sciences) version 20 and transferred to Microsoft word 2016. Numerical data (zones of inhibition) was expressed by mean and standard deviations, while categorical data (sources of isolates, age groups, gender, clinical settings, species, antifungal sensitivity and resistance patterns of isolates) were stated in frequency and percentages and their associations were tested by applying Chi-square test. P-value $<0.05$ considered significant. 


\section{Results}

\subsection{Demography and Sensitivity Pattern of Candidiasis}

\subsubsection{Number of Samples and Distribution of Candidiasis within Different Age Groups and Gender}

From $1^{\text {st }}$ March 2018 to $30^{\text {th }}$ May 2018, 130 (12.75\%) samples were found to be candida positive out of the total 1020. The age groups of patients reported in this study with candidal infections ranged from 2 months to 89 year with mean age of $51 \pm 20.27$ year. The most candidal infected age group was 51 to 60 year with an overall prevalence of $26.20 \%$ (Figure 1). Among males the most frequently candidal infected age group reported was ranged between 51 - 60 year of age whereas among females from 31 - 40 year with highly significant $\mathrm{p}$ value $<0.01$ (Table 1).

\subsubsection{Distribution of Clinical Settings and Basic Demography of Candidal Patients}

As shown in Table 2, majority (40\%) of the candida positive samples were isolated from ICU patients followed by patients admitted in wards and private rooms (36.2\%). It was observed that females were predominantly 80 (61.54\%) infected with candidiasis in comparison to the males (Figure 2). In females candida positive samples were frequently associated with candidal vaginitis 35 $(43.75 \%)$ in comparison to males whose candidal positive samples were mostly related to candidal infected tracheal aspirates 14 (28\%) with highly significant $\mathrm{P}$ value $(<0.01)$. Overall in both gender, candidiasis was frequently retrieved from vagina (26.9\%) sputum (19.23\%) and urine (17.7\%). Candidal specimens in both genders are displayed in Table 3.

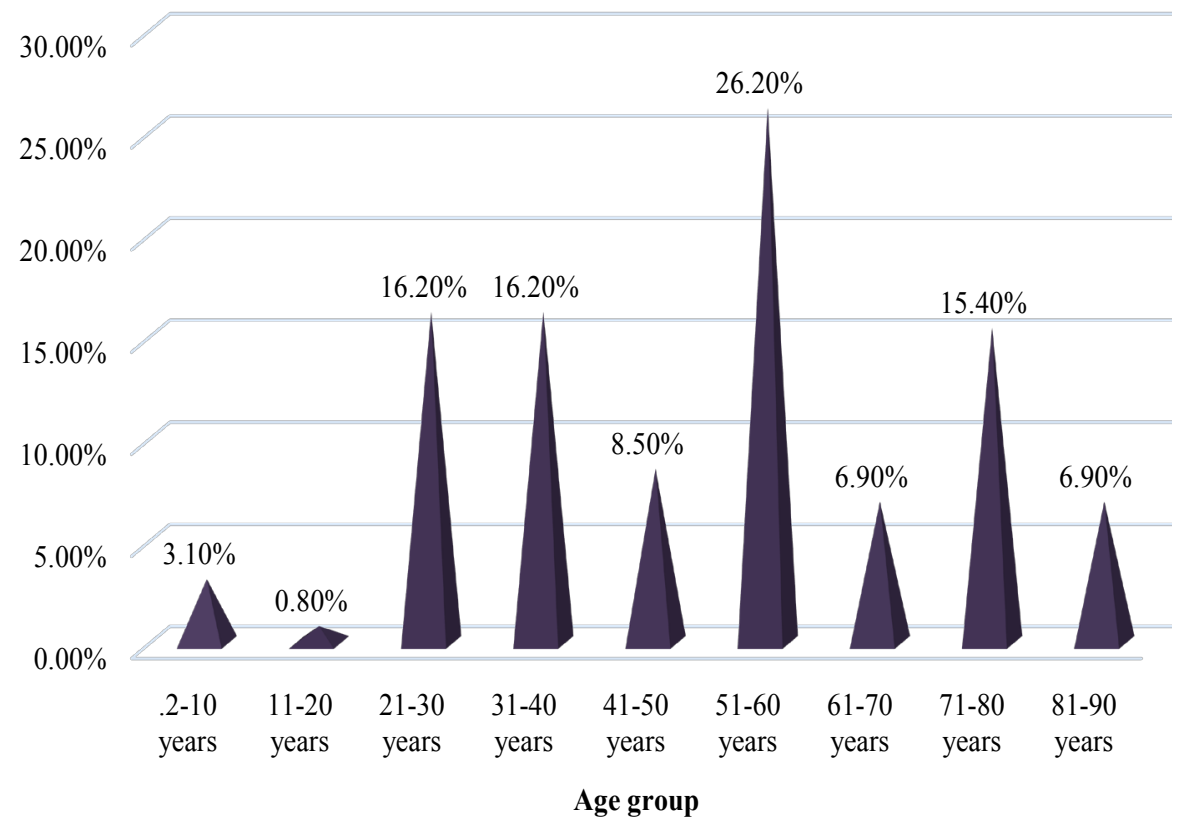

Figure 1. Frequency of candidal infections among various age groups. 


\section{GENDER DISTRIBUTION}

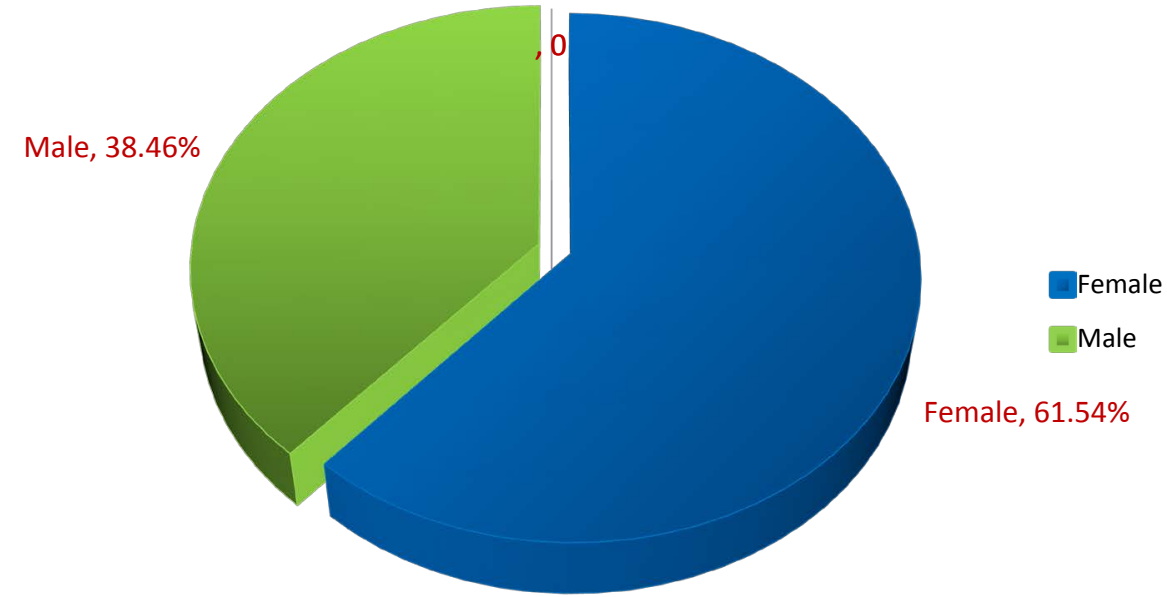

Figure 2. Frequency of gender distribution in isolated cases of candidiasis.

Table 1. Association of candidal patients' age group with their gender.

\begin{tabular}{|c|c|c|c|c|}
\hline \multirow{3}{*}{ Age group } & \multicolumn{2}{|c|}{ Gender } & \multirow{2}{*}{$\begin{array}{c}\text { Total no. of gender in } \\
\text { age groups }\end{array}$} & \multirow{3}{*}{$P$ - value } \\
\hline & $\begin{array}{c}\text { Male } \\
50(38.48 \%)\end{array}$ & $\begin{array}{c}\text { Female } \\
80(61.5 \%)\end{array}$ & & \\
\hline & n (\%) & n (\%) & n (\%) & \\
\hline $0.2-10$ years & $3(8)$ & $0(0)$ & $3(3.1)$ & \multirow{10}{*}{$<0.01$} \\
\hline $11-20$ years & $1(2)$ & $0(0)$ & $1(0.8)$ & \\
\hline 21 - 30 years & $3(6)$ & $18(22.5)$ & $21(16.2)$ & \\
\hline $31-40$ years & $2(4)$ & $19(23.8)$ & $21(16.2)$ & \\
\hline 41 - 50 years & $0(0)$ & $11(13.8)$ & $11(8.5)$ & \\
\hline 51 - 60 years & $16(32)$ & $18(22.5)$ & $34(26.2)$ & \\
\hline $61-70$ years & $4(8)$ & $5(6.2)$ & $9(6.9)$ & \\
\hline $71-80$ years & $14(28)$ & $6(7.5)$ & $20(15.4)$ & \\
\hline 81 - 90 years & $6(12)$ & $3(3.8)$ & $9(6.9)$ & \\
\hline Total & $50(100)$ & $80(100)$ & $130(100)$ & \\
\hline
\end{tabular}

Table 2. Overall frequency of number of candidal cases from different settings of Ziauddin group of hospitals in different regions of Karachi.

\begin{tabular}{cccc}
\hline Clinical settings & Frequency (n) & Percent (\%) & Total (\%) \\
\hline OPD & 31 & 23.8 & 23.8 \\
IPD - ICU/CCU/E.R/O.T & 52 & 40.0 & \\
IPD - WARD/ADMIT/PVT ROOM & 47 & 36.2 & 10.2 \\
Total & 130 & 100 & 100 \\
\hline
\end{tabular}

OPD—out patient department, IPD—in patient department, ICU_intensive care unit, CCU—critical care unit, ER-emergency department, OT_-operation theatre, PVT ROOM-private room. 
Table 3. Association of Gender with isolated candidal sources by Pearson Chi-Square.

\begin{tabular}{ccccc}
\hline & \multicolumn{4}{c}{ Gender } \\
\cline { 2 - 4 } Source of isolates & Male (38.46\%) & Female (61.54\%) & P - value \\
\cline { 2 - 4 } & $\mathrm{n}$ & $\%$ & $\mathrm{n}$ & $\%$ \\
\hline Ascitic fluid 1 (0.8\%) & 1 & 2 & 0 & 0 \\
Blood 8 (6.2\%) & 5 & 10 & 3 & 3.75 \\
HVS* 35 (26.9\%) & 0 & 0 & 35 & 43.75 \\
N/G tube** 1 (0.8\%) & 0 & 0 & 1 & 1.25 \\
Peritoneal fluid 1 (0.8\%) & 1 & 2 & 0 & 0 \\
Pus 9 (6.9\%) & 9 & 18 & 0 & 0 \\
Sputum 25 (19.23\%) & 11 & 22 & 14 & 17.5 \\
Tip 6 (4.6\%) & 0 & 0 & 6 & 7.5 \\
Tracheal aspirate 17 (13.1\%) & 14 & 28 & 3 & 3.75 \\
Urine 23 (17.7\%) & 9 & 18 & 14 & 17.5 \\
Wound swab 4 (3.1\%) & 0 & 0 & 4 & 5 \\
Total 130 (100\%) & 50 & 100 & 80 & 100 \\
\hline
\end{tabular}

${ }^{\star}$ HVS: High vaginal swab, ${ }^{* *}$ N/G: Nasogastric tube.

\subsubsection{Frequency of Candidal Species}

Among all candidal species, $C$. albicans was the most frequent specie isolated from $80 \%$ of the candida positive samples while among non albicans the isolated species were C. tropicalis $10 \%$, C. glabrata $9.23 \%$ and C. ciferrii $0.8 \%$. Overall $25.40 \%$ isolates showed co-infection with bacteria, as shown in Figure 3.

\subsubsection{Sensitivity and Resistance Patterns of Clinical Isolates}

Table 4 demonstrates the sensitivity and resistance patterns of identified candidal isolates against fluconazole and voriconazole. According to our results voriconazole was found to be more resistant $(70.8 \%)$ in comparison to fluconazole showing resistance of $57.7 \%$ with overall resistance to both azoles was $64.25 \%$ (35.75\% sensitivity). Though the cross resistance detected was (66) $50.7 \%$.

\subsection{Candidemia-A Life Threatening Invasive Blood Stream Infection}

In present study out of 130 candidal positive isolates 8 specimens were obtained from blood as source of infection at a rate of $6.15 \%$. Candidemia was observed to be $62.5 \%$ common in males in comparison to females (Table 5). Among species C. non albicans were the major offender for this blood stream infection involving C. tropicalis $87.5 \%$ and C. ciferrii $12.5 \%$ (Table 6). While mortality rate analyzed was $50 \%$ (Table 5). Among species $87.5 \%$ of samples showed C. tropicalis predominance while overall sensitivity profile against both azoles showed 75\% resistance, displayed in Table 7. 


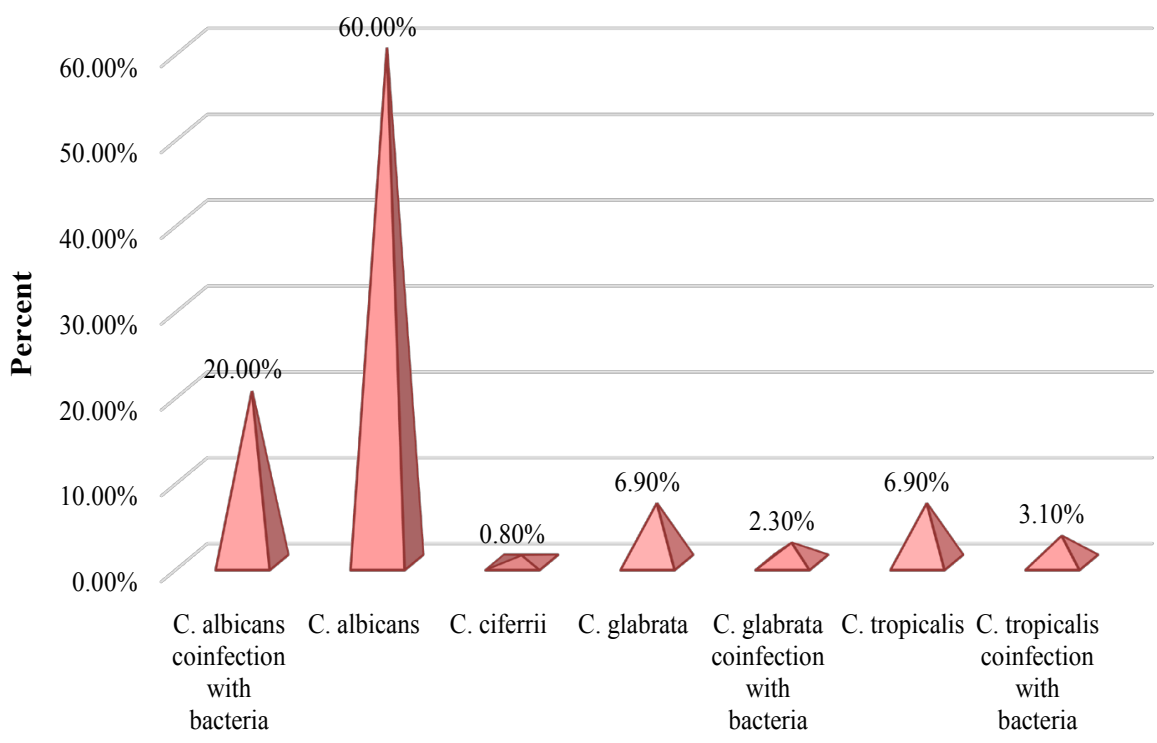

\section{Candidal species}

Figure 3. Bar chart showing frequency of Candidal species.

Table 4. Over all Sensitivity and resistance of clinical isolates to antifungal drugs.

\begin{tabular}{ccccccc}
\hline \multirow{2}{*}{$\begin{array}{c}\text { Sensitivity } \\
\text { pattern }\end{array}$} & \multicolumn{2}{c}{ Fluconazole } & \multicolumn{2}{c}{ Voriconazole } & \multicolumn{2}{c}{ Cross Resistance } \\
\cline { 2 - 6 } & $\mathbf{n}$ & $\%$ & $\mathbf{n}$ & $\%$ & $\mathbf{n}$ & $\%$ \\
\hline Sensitive & 55 & 42.3 & 38 & 29.2 & & \\
Resistant & 75 & 57.7 & 92 & 70.8 & 66 & 50.7 \\
Total & 130 & 100 & 130 & 100 & & \\
\hline
\end{tabular}

Where, inhiboitry zone $>14 \mathrm{~mm}$ of fluconazole $(25 \mathrm{mcg})$ and $>17 \mathrm{~mm}$ of voriconazole $(1 \mathrm{mcg})$ were considered sensitive [11] [12] [13].

Table 5. Distribution of death rate with gender in candidemia patients.

\begin{tabular}{|c|c|c|c|c|c|}
\hline \multirow{3}{*}{ Gender } & \multicolumn{4}{|c|}{ Candidemia patients } & \multirow{3}{*}{$P$ value } \\
\hline & \multicolumn{2}{|c|}{ Alive $4(50 \%)$} & \multicolumn{2}{|c|}{ Expired $4(50 \%)$} & \\
\hline & $\mathrm{n}$ & $\%$ & $\mathrm{n}$ & $\%$ & \\
\hline Male $5(62.5 \%)$ & 1 & 20 & 4 & 80 & \\
\hline Female $3(37.5 \%)$ & 3 & 100 & 0 & 0 & $0.028^{\star}$ \\
\hline
\end{tabular}

${ }^{*} \mathrm{p}$ value is significant $(<0.05)$.

Table 6. Distribution of candidal species responsible for candidemia.

\begin{tabular}{ccc}
\hline Candidal species & Frequency (n) & Percent (\%) \\
\hline C. ciferrii & 1 & 12.5 \\
C. tropicalis & 7 & 87.5 \\
Total & 8 & 100.0 \\
\hline
\end{tabular}


Table 7. Antifungal sensitivity profile of candidemia isolates.

\begin{tabular}{ccccc}
\hline \multirow{2}{*}{ Sensitivity pattern } & \multicolumn{2}{c}{ Fluconazole } & \multicolumn{2}{c}{ Voriconazole } \\
\cline { 2 - 5 } & $\mathbf{n}$ & $\%$ & $\mathbf{n}$ & $\%$ \\
\hline Sensitive & 3 & 37.5 & 1 & 12.5 \\
Resistant & 5 & 62.5 & 7 & 87.5 \\
Total & 8 & 100 & 8 & 100 \\
\hline
\end{tabular}

Where, inhiboitry zone $>14 \mathrm{~mm}$ of fluconazole $(25 \mathrm{mcg})$ and $>17 \mathrm{~mm}$ of voriconazole $(1 \mathrm{mcg})$ were considered sensitive [11] [12] [13].

\section{Discussion}

Global rise in the occurrence of opportunistic fungal infections is exacerbating and has been quite challenging for the medical researchers to fix this issue with the development of new generation of antifungal agents. Since many of the currently available antifungal drugs have undesirable side effects, are ineffective against new or reemerging fungi and may lead to the rapid development of resistance. Furthermore, the improvement of new antifungal compounds is limited due to the eukaryotic architecture of fungal cell, which is very similar to the human cells, and has huge genome's plasticity [14] [15]. The knowledge of incidence and prevalence rate along with the resistance patterns of candidiasis in our country is lacking at national level which has a direct influence on treating these patients with the most appropriate antifungal drug [16]. Furthermore, antifungal susceptibility testing is not applied routinely as a standard protocol in most of the microbiological laboratories of Pakistan as the $\mathrm{C} / \mathrm{S}$ testing is quite expensive, costs around 20 US $\$$ (dollars) that cannot be managed by majority of our low socioeconomic population [5].

Candidal patients notified in our study were from 2 month to 89 year of ages. Most of these patients had weak immunity, comorbidity, long term antibiotic treatments and hospitalization. Globally candidiasis is known as the disease of people at extreme of ages, usually occurs at the ages of less than 1 and greater than 70 year, particularly, due to their compromised immunity [17]. However, a study conducted in a tertiary setting of Rawalpindi, Pakistan showed candidiasis commonest in the age group of 21 - 40 year [5]. In comparison, most of our study population was from $51-60$ year age group with mean age of $51 \pm 20.27$ year (Table 1). Two other local studies showed its high frequency in people at 50 year and another study at $>60$ year of ages [18] [19].

According to Figure 2, higher frequency of candidal isolates belonged to females, being $61.54 \%$ with female to male ratio of $8: 5$. Our results are parallel to the findings of two studies conducted in other cities of Pakistan (Islamabad and Rawalpindi), showed candidaiasis predominantly in females (56\% and 65.8\%) [5] [17]. While contrary to our results, Bhattacharjee et al., India, reported males (63.32\%) more frequently infected with candidiasis than females (36.38\%) [20].

In this study candidiasis was commonly isolated from vagina (26.9\%) followed by sputum (19.23\%), urine (17.7\%) and tracheal aspirates (13.1\%) as 
mentioned in Table 3. In comparison to our results a study conducted in Korea reported isolates commonly from urine 62.4\% [21]. Candida is a common resident in areas of human body which are exposed to the environment therefore more prone to infect under advantageous circumstances as the patients' immunity decline, prolonged catheterization and pregnancy. Additionally, female reproductive hormones have receptors for candida due to which these organisms colonize and infect female genitalia (mainly vagina) [18] [22]. This is the reason why candidias was mainly isolated from females in our study.

In our study, majority of the candida positive samples were isolated from ICU patients with $40 \%$ frequency followed by patients admitted in wards and private rooms $(36.2 \%)$ which is contradictory to the findings of Aslam et al., Pakistan showed 52\% of isolates, predominantly from Medical unit [5] and Noor et al., Malaysia documented 25\% from wards [23]. Previously it has been documented that Candidal strains are considered to be $80 \%$ responsible pathogenic opportunistic organism among hospital based fungal infections [20]. Protracted hospitalization is associated with increased risk of acquiring candida infections as these patients usually hospitalized for their co-morbids, had already poor health and weakened immune system [9].

In this study the principal pathogenic specie responsible for candidiasis in general are $C$. albicans $80 \%$ followed by $C$. tropicalis $10 \%, C$. glabrata $9.23 \%$ and C. ciferrii $0.8 \%$ (Table 3 ). These findings are in accordance to a global surveillance study ARTEMIS conducted by Pfaller, expressed $C$. albicans as the main culprit among all other species with overall 57.7\% prevalence internationally, after that $C$. glabrata $14.8 \%$ and $C$. tropicalis $9.4 \%$ were amongst the prevalent candida species [24]. Our results are also corresponding to a study of China by Park et al. showed C. albicans 64\%, C. tropicalis $18.1 \%$ and C. glabrata $8.6 \%$ [16] [25]. While contrasting with the results of an Indian study which showed majority $(63.3 \%)$ of the isolates were non albicans and the commonest isolated specie was Candida tropicalis (41.1\%), followed by Candida albicans (36.7\%) and Candida glabrata (10\%) [26]. Considerable discrepancy has been observed in the frequency of Candidal species distribution among all candida positive cases across the world but the actual reason is still unknown [10].

Azoles are one of the most commonly prescribed antifungal drugs for both systemic and topical fungal infections and are administered by oral and topical routes .These are frequently over the counter (OTC) available drugs, mainly fluconazole in pharmacies since $19^{\text {th }}$ century and their constant rising resistance is becoming a big threat globally [27] [28] [29]. According to a global surveillance study, conducted from the year 2001 to 2007 resistance to fluconazole reported $13.8 \%$ and voriconazole $6 \%$ [10]. While this current study displayed overall resistance of $64.25 \%$ for both of antifungals, fluconazole and voriconazole while individual resistance for fluconazole was $57.7 \%$ and for voriconazole was $70.8 \%$ (Table 4), in contrast a study conducted in Rawalpindi, Pakistan showed overall resistance of both antifungals, $46 \%$ whereas, respective resistance to fluconazole 
and voriconazole was $85 \%$ and $4 \%$ [30]. This shows a drastic variation in resistance patterns of widely prescribed, azoles in both cities of Pakistan, Karachi and Rawalpindi. The emergence of resistance to voriconazole is alarming as it is used as an alternative to fluconazole and itraconazole which may represent its irrational use more than fluconazole in our clinical settings. In our study cross resistance to both azoles observed was 66 (50.7\%) among all isolates. While according to the previous studies conducted in various countries including Pakistan, Brazil and India, cross resistance between these antifungal drugs reported in a range of $34 \%$, to $74 \%$ [18] [30] [31] [32], which is due to their analogous chemical structure [33].

\section{Candidemia}

Candidal infection in blood is a big life threat for the affected individuals. In this present study it is reported $6.15 \%$ which is resembling to the report of CDC (first global report published on candidemia) In this study, candidemia was found to be fairly prevalent in male gender $62.5 \%$. The overall mortality rate observed was $50 \%$, while in earlier studies death rate was found to be approximately $26 \%$ to 52\% [34] [35] [36] [37]. According to reports of ARTEMIS and SENTRY, globally $38 \%$ to $70 \%$ candidemia occurs due to C. albicans among all species since centuries but now this rate is shifting towards non albicans [38] which is also obvious from our study, showing Non albicans $100 \%$ responsible for candidemia. These non albicans species in this study are C. tropicalis $87.5 \%$ as the main culprit of candidemia followed by $C$. ciferrii $12.5 \%$. A study conducted in same country showed $C$. tropicalis 33\% responsible specie for candidemia [39]. Unexpectedly, in our study a rare specie, $C$. ciferrii was isolated in a patient with blood stream infection. As documented, it is an infrequent candidal specie, known for its superficial pathogenicity in the field of microbiology [40]. This is in contrast to our study displaying C. ciferrii causing blood stream infection, which illustrates that any superficial candidal specie could invade systemically when person get immunocompromised which is also true for our study sample. Although our sample size for candidemia was small but these results verified the results of previous studies to consider $C$. tropicalis as the main culprit in cases of candidemia in our clinical set up. Our results are also in agreement with the previous researches showing non-candida albicans as the main source of invasive candidiasis [41].

In our study, for candidemia both antifungals were highly resistant but fluconazole showed more (37.5\%) sensitivity in comparison to voriconazole $(12.5 \%)$ (Table 5), among which C. tropicalis was $71.4 \%$ resistant to fluconazole and $100 \%$ to voriconazole. The global study ARTEMIS conducted from 1997 to 2003 showed resistance of $C$. tropicalis against fluconazole from $3 \%$ to $6.6 \%$ [15], which is quite low as compared to our results. Surprisingly our results were contradictory to our local data conducted in 2013, according to which the most resistant specie was C. krusei $100 \%$, followed by and C. glabrata 15\% [39]. 


\section{Conclusions}

Candidiasis is highly prevalent in our clinical set up and more frequently invading females in comparison to males as most of the positive isolates were retrieved from HVS (high vaginal swabs), while C. albicans was still found to be the most prevalent isolated specie among all positive candidal samples. We didn't find the shift towards non albicans except in candidemia cases where absolutely non albicans were the main offenders. Amazingly a rare candidal specie C. ciferrii, known for superficial candidiasis was reported in our study as a cause of systemic (nosocomial) blood infection.

Our study also demonstrated that the resistance of the most commonly prescribed antifungals, azoles have shown a rapid rise. Therefore, it is recommended that before prescription of antifungal drugs the clinicians should routinely recommend culture and sensitivity testing of samples taken from candida infected individuals. This will help in prescription of more effective and appropriate antifungal drugs which will lower the development of their resistance in our clinical set up. Subsequently the effective antifungal therapy will reduce the repeated use of antifungal drugs, with decreased frequency of adverse effects, improvement in over health and cut down the cost of antifungal therapy on the pockets of the candidal infected patients. Consequently, there is an intense urge to introduce novel antifungals with better efficacy, less side effects, and should be affordable to the patients for prolonged therapy. Additionally, preventive measures should be taken to decrease the incidence of nosocomial candidiasis.

\section{Limitations of the Study}

In this study we tested the sensitivity patterns of only two commonly prescribed antifungals due to limited budget. Additionally, the sampling was conducted only in one major city of Pakistan, Karachi and could not include fungal infected patients of other regions, also because of the limited budget, and lack of access to their clinical set ups.

\section{Conflicts of Interest}

The authors declare no conflicts of interest regarding the publication of this paper.

\section{References}

[1] Brown, G.D., Denning, D.W., Gow, N.A., Levitz, S.M., Netea, M.G. and White, T.C. (2012) Hidden Killers: Human Fungal Infections. Science Translational Medicine, 4, 165rv113-165rv113. https://doi.org/10.1126/scitranslmed.3004404

[2] Ng, K.P., Kuan, C.S., Kaur, H., Na, S.L., Atiya, N. and Velayuthan, R.D. (2015) Candida Species Epidemiology 2000-2013: A Laboratory-Based Report. Tropical Medicine \& International Health, 20, 1447-1453. https://doi.org/10.1111/tmi.12577

[3] Meiller, T.F., Hube, B., Schild, L., Shirtliff, M.E., Scheper, M.A., Winkler, R. and Jabra-Rizk, M.A. (2009) A Novel Immune Evasion Strategy of Candida albicans. Proteolytic Cleavage of a Salivary Antimicrobial Peptide. PloS One, 4, e5039. 
https://doi.org/10.1371/journal.pone.0005039

[4] Dominguez, E.G. and Andes, D.R. (2017) Candida Biofilm Tolerance: Comparison of Planktonic and Biofilm Resistance Mechanisms. In: Candida albicans: Cellular and Molecular Biology, Springer, Berlin, 77-92.

https://doi.org/10.1007/978-3-319-50409-4_6

[5] Aslam, A., Akhtar, N., Hasan, F. and Shah, A.A. (2015) Prevalence and in Vitro Antifungal Susceptibility Pattern of Candida Species in a Tertiary Care Hospital, Rawalpindi, Pakistan. Pakistan Journal of Zoology, 47, 335-342.

[6] Rathod, T., Padalia, H. and Chanda, S. (2015) The Potential of Plant Extracts against Multidrug Resistant Candida Species-A Review. Gujarat. http://www.microbiology5.org/microbiology5/book/246-256.pdf

[7] Garcia-Solache, M.A. and Casadevall, A. (2010) Global Warming Will Bring New Fungal Diseases for Mammals. MBio, 1, e00061-10. https://doi.org/10.1128/mBio.00061-10

[8] Salma, S., Shah, M.A. and Rehman, S. (2012) Rainfall Trends in Different Climate Zones of Pakistan. Pakistan Journal of Meteorology, 9, 37-47.

[9] Sardi, J., Scorzoni, L., Bernardi, T., Fusco-Almeida, A. and Giannini, M.M. (2013) Candida Species: Current Epidemiology, Pathogenicity, Biofilm Formation, Natural Antifungal Products and New Therapeutic Options. Journal of Medical Microbiology, 62, 10-24. https://doi.org/10.1099/jmm.0.045054-0

[10] Pfaller, M., Diekema, D., Gibbs, D., Newell, V., Ellis, D., Tullio, V. and Group, G.A. S. (2010) Results from the ARTEMIS DISK Global Antifungal Surveillance Study, 1997 to 2007: A 10.5-Year Analysis of Susceptibilities of Candida Species to Fluconazole and Voriconazole as Determined by CLSI Standardized Disk Diffusion. Journal of Clinical Microbiology, 48, 1366-1377. https://doi.org/10.1128/JCM.02117-09

[11] Pfaller, M.A., Castanheira, M., Messer, S.A. and Jones, R.N. (2015) In Vitro Antifungal Susceptibilities of Isolates of Candida spp. and Aspergillus spp. from China to Nine Systemically Active Antifungal Agents: Data from the SENTRY Antifungal Surveillance Program, 2010 through 2012. Mycoses, 58, 209-214. https://doi.org/10.1111/myc.12299

[12] Wayne, P.A. (2009) Clinical and Laboratory Standards Institute (CLSI). Method for Antifungal Disk Diffusion Susceptibility Testing of Yeasts, Approved Guideline-2nd Edition, CLSI Document M44-A2, New York.

[13] Wayne, P.A. (2009) Zone Diameter Interpretive Standards, Corresponding Minimal Inhibitory Concentration (MIC) Interpretive Breakpoints, and Quality Control Limits for Antifungal Disk Diffusion Susceptibility Testing of Yeasts. Third International Supplement CLSI document-M444-S3, New York.

[14] Carmona, E.M. and Limper, A.H. (2017) Overview of Treatment Approaches for Fungal Infections. Clinics in Chest Medicine, 38, 393-402. https://doi.org/10.1016/j.ccm.2017.04.003

[15] Branquinha, M.H. and Kneip, L.F. (2017) Fungal Infections: A Veritable Worldwide Problem. Journal of Infectious Diseases and Medical Microbiology, 1, 1-2.

[16] Kumar, S., Vyas, A., Kumar, M. and Mehra, S. (2013) Application of Chromagar Candida for Identification of Clinically Important Candida Species and Their Antifungal Susceptibility Pattern. International Journal of Biological and Medical Research, 4, 3600-3606.

[17] Pfaller, M.A. and Diekema, D.J. (2007) Epidemiology of Invasive Candidiasis: A Persistent Public Health Problem. Clinical Microbiology Reviews, 20, 133-163. 
https://doi.org/10.1128/CMR.00029-06

[18] Tasneem, U., Siddiqui, M.T., Faryal, R. and Shah, A.A. (2017) Prevalence and Antifungal Susceptibility of Candida Species in a Tertiary Care Hospital in Islamabad, Pakistan. The Journal of the Pakistan Medical Association, 67, 986-991.

[19] Samyuktha, A.A. and Saikumar, C. (2017) Isolation, Identification and Speciation of Candida Species from Various Clinical Specimens in a Tertiary Care Hospital in Chennai. Scholars Journal of Applied Medical Sciences, 5, 3460-3468.

[20] Bhattacharjee, P. (2016) Epidemiology and Antifungal Susceptibility of Candida Species in a Tertiary Care Hospital, Kolkata, India. Current Medical Mycology, 2, 20-27. https://doi.org/10.18869/acadpub.cmm.2.2.5

[21] Kim, G.Y., Jeon, J.S. and Kim, J.K. (2016) Isolation Frequency Characteristics of Candida Species from Clinical Specimens. Mycobiology, 44, 99-104. https://doi.org/10.5941/MYCO.2016.44.2.99

[22] Rashwan, N.M., Mohamed, A.K.A., El-Deen, S.S., Ahmed, E.H. and Ismail, S.A. (2010) Pattern of Candida Urinary Tract Infections among Cancer Patients in South Egypt Cancer Institute. Bulletin of Pharmaceutical Sciences, 33, 121-130.

[23] Noor, S.S.M. (2008) Epidemiology of Candida Species in Tertiary-Teaching Hospital in Malaysia. International Medical Journal, 15, 291-294.

[24] Pfaller, M.A., Diekema, D.J., Messer, S.A., Boyken, L. and Hollis, R.J. (2003) Activities of Fluconazole and Voriconazole against 1,586 Recent Clinical Isolates of Candida Species Determined by Broth Microdilution, Disk Diffusion, and Etest Methods: Report from the ARTEMIS Global Antifungal Susceptibility Program, 2001. Journal of Clinical Microbiology, 41, 1440-1446. https://doi.org/10.1128/JCM.41.4.1440-1446.2003

[25] Park, G., Kim, S., Kang, H., Lee, H., Jo, H., Jeoung, H., Chang, K., et al. (2016) Isolation Frequency and Epidemiological Characterization of Yeast-Like Fungi and Co-Infected Bacteria Isolated from Clinical Patient. Journal of Yeast and Fungal Research, 7, 7-18. https://doi.org/10.5897/JYFR2015.0160

[26] Kaur, R., Dhakad, M.S., Goyal, R. and Kumar, R. (2016) Emergence of Non-Albicans Candida Species and Antifungal Resistance in Intensive Care Unit Patients. Asian Pacific Journal of Tropical Biomedicine, 6, 455-460. https://doi.org/10.1016/j.apjtb.2015.12.019

[27] Mårdh, P.A., Wågström, J., Landgren, M. and Holmén, J. (2004) Usage of Antifungal Drugs for Therapy of Genital Candida Infections, Purchased as Over-the-Counter Products or by Prescription: 1. Analysis of a Unique Database. Infectious Diseases in Obstetrics and Gynecology, 12, 91-97. https://doi.org/10.1080/10647440400003873

[28] Kontoyiannis, D.P. (2017) Antifungal Resistance: An Emerging Reality and a Global Challenge. The Journal of Infectious Diseases, 216, S431-S435. https://doi.org/10.1093/infdis/jix179

[29] Steib-Bauert, M., Knoth, H., Dörje, F., Strehl, E., Rothe, U., Maier, L. and Kern, W.V. (2005) Hospital Use of Systemic Antifungal Drugs. BMC Clinical Pharmacology, 5, 1. https://doi.org/10.1186/1472-6904-5-1

[30] Jamil, B., Bokhari, M.T.M., Saeed, A., Bokhari, M.Z.M., Hussain, Z., Khalid, T., Abbasi, S.A., et al. (2017) Candidiasis: Prevalence and Resistance Profiling in a Tertiary Care Hospital of Pakistan. JPMA. The Journal of the Pakistan Medical Association, 67, 688 .

[31] Mane, A., Vidhate, P., Kusro, C., Waman, V., Saxena, V., Kulkarni-Kale, U. and 
Risbud, A. (2016) Molecular Mechanisms Associated with Fluconazole Resistance in Clinical Candida Albicans Isolates from India. Mycoses, 59, 93-100.

https://doi.org/10.1111/myc.12439

[32] Wiebusch, L., Lonchiati, D., Rodrigues, L., Dantas, C., Almeida, A. and Oliveira, K. (2014) Profile Susceptibility to Fluconazole and Voriconazole Antifungals by Species of Candida albicans Isolated from Urine Culture. BMC Proceedings, 8, 34. https://doi.org/10.1186/1753-6561-8-S4-P34

[33] Peyton, L.R., Gallagher, S. and Hashemzadeh, M. (2015) Triazole Antifungals: A Review. Drugs Today, 51, 705-718.

[34] Hirano, R., Sakamoto, Y., Kudo, K. and Ohnishi, M. (2015) Retrospective Analysis of Mortality and Candida Isolates of 75 Patients with Candidemia: A Single Hospital Experience. Infection and Drug Resistance, 8, 199. https://doi.org/10.2147/IDR.S80677

[35] Andes, D.R., Safdar, N., Baddley, J.W., Playford, G., Reboli, A.C., Rex, J.H. and Kullberg, B.J. (2012) Impact of Treatment Strategy on Outcomes in Patients with Candidemia and Other Forms of Invasive Candidiasis: A Patient-Level Quantitative Review of Randomized Trials. Clinical Infectious Diseases, 54, 1110-1122. https://doi.org/10.1093/cid/cis021

[36] Arendrup, M.C. (2013) Candida and Candidaemia. Susceptibility and Epidemiology. Danish Medical Journal, 60, B4698.

[37] de Molina, F.J.G., León, C., Ruiz-Santana, S. and Saavedra, P. (2012) Assessment of Candidemia-Attributable Mortality in Critically Ill Patients Using Propensity Score Matching Analysis. Critical Care, 16, R105. https://doi.org/10.1186/cc11388

[38] Yapar, N. (2014) Epidemiology and Risk Factors for Invasive Candidiasis. Therapeutics and Clinical Risk Management, 10, 95-105.

https://doi.org/10.2147/TCRM.S40160

[39] Farooqi, J., Jabeen, K., Saeed, N., Iqbal, N., Malik, B., Lockhart, S., Hasan, R., et al. (2013) Invasive Candidiasis in Pakistan: Clinical Characteristics, Species Distribution and Antifungal Susceptibility. Journal of Medical Microbiology, 62, 259-268. https://doi.org/10.1099/jmm.0.048785-0

[40] Villanueva-Lozano, H., Treviño-Rangel, R.D.J., Hernández-Balboa, C.L., González, G.M. and Martínez-Reséndez, M.F. (2016) An Unusual Case of Candida Ciferrii Fungemia in an Immunocompromised Patient with Crohn's and Mycobacterium Bovis Disease. The Journal of Infection in Developing Countries, 10, 1156-1158. https://doi.org/10.3855/jidc.8228

[41] Kullberg, B.J. and Arendrup, M.C. (2015) Invasive Candidiasis. New England Journal of Medicine, 373, 1445-1456. https://doi.org/10.1056/NEJMra1315399 